\title{
Improved Control Strategy of Interlinking Converters With Synchronous Generator Characteristic in Islanded Hybrid AC/DC Microgrid
}

\author{
Gongxin Qi, Alian Chen, and Jie Chen
}

\begin{abstract}
In this paper, an improved control strategy of interlinking converters for hybrid AC/DC microgrid operated in islanding mode is proposed, which applies synchronous generator model to the converters. This enhanced scheme adopts direct frequency control method to realize active power sharing and improves the transient frequency stability by using synchronverter technology. Unlike existing droop control methods of interlinking converters that mostly just focus on power sharing, this scheme can not only maintain proportional power distributed between $\mathrm{DC}$ and $\mathrm{AC}$ subgrid, but also regulate the $\mathrm{AC}$ subgrid voltage directly to improve its poor frequency stability during ACside loading transitions in autonomous operation. It's noteworthy that this scheme can also keep the AC-side loads working uninterruptedly during AC subgrid faults events by using voltage-controlled method. Moreover, any additional energy storage or inverters are not required to assist interlinking converters for microgrid frequency regulation. The effectiveness of this modified control method is verified by offline time-domain simulation and real-time experiment in MATLAB/Simulink and OPAL-RT digital platform respectively.
\end{abstract}

Index Terms - Frequency transient stability, hybrid AC/DC microgrid, interlinking converter, proportional power sharing, sychronverter.

\section{INTRODUCTION}

$\mathrm{N}$ OWADAYS, with the rapid increase of photovoltaic, fuel cells, energy storages and many other DC energy power systems in microgrids as well as modern DC loads such as electric vehicles, and considering the existing century-long AC power systems, hybrid AC/DC microgrids have obtained more and more attention [1]. In a hybrid microgrid, as shown in Fig. 1, AC and DC microgrids are interconnected together via interlinking converters (ILCs), making it possible to exchange power among all distributed generations (DGs). Many types of distributed resources are connected to $\mathrm{DC}$ and $\mathrm{AC}$ buses by different power converters. Once there are some faults in utility grid, the static switch at point of common coupling (PCC) will be kept open and the hybrid microgrid works in an islanded mode. It is noteworthy that

Manuscript received June 15, 2017. This work was supported by National Natural Science Foundation of China under Grant 51377101.

The authors are with the School of Control Science and Engineering, Shandong University, Jinan 250061, China (e-mail: qigongxin@mail.sdu. edu.cn; chenalian@sdu.edu.cn; 1457935436@qq.com).

Digital Object Identifier 10.24295/CPSSTPEA.2017.00015
ILCs control method plays an important part in the hybrid microgrids especially without the support of utility grid, coordinating the dual subsystems to regulate $\mathrm{AC}$ and $\mathrm{DC}$ bus voltages and exchange power proportionally between $\mathrm{DC}$ subgrid and AC subgrid [2].

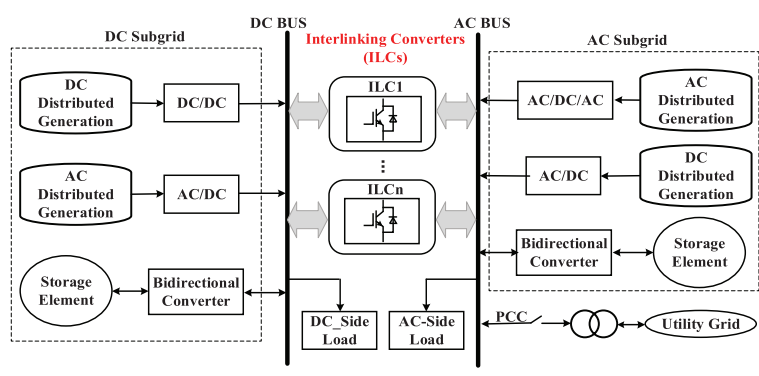

Fig. 1. Structure of hybrid AC/DC microgrid.

All of the ILCs control methods can be classified into communication-based and communication-less strategies. References [3]-[5] present centralized control schemes based on energy management system to manage power flows of a hybrid microgrid and regulate the $\mathrm{AC}$ and $\mathrm{DC}$ bus voltages on their reference values. However, these methods highly depend on fast and accurate communication system. But any link malfunctions or communication interference would likely lead to instability of the whole system. So communication-based control methods are usually viewed as less reliable and they are also not suitable to be applied to multiple parallel ILCs due to many wire links [6]. Therefore, communication-less control based on the classical droop operating principles is preferred.

Many wireless control methods based on normalized droop control have been proposed in recent literatures. In [7]-[11], it has been suggested to just measure per-unit values of the AC subgrid frequency and DC subgrid voltage to determine the reference power for ILCs and consequently balance supply-demand between two subgrids. Besides, multiple ILCs could be used in parallel to enhance capacity and reliability without wire links. However, these schemes employed in ILCs usually use frequency variation and current-controlled method. Large frequency variation has bad effects on AC-side frequency stability and would lead to poor power quality. Besides, the current-controlled method doesn't offer voltage support for AC bus and even deteriorates $\mathrm{AC}$-side frequency stability while transferring 
exchanged power. Especially during the AC-side loads transients, microgrid frequency could oscillate heavily and even beyond allowable limits.

Motivated by aforementioned problems, this paper proposes a modified method to optimize AC subgrid frequency stability based on normalized droop control method. The direct frequency control scheme is adopted to manage proportional power flows and enhance frequency dynamic response meanwhile. Furthermore, synchronverter technology is applied to ILCs control system to improve poor dynamic frequency stability by mimicking a synchronous generator. Virtual inertia can make ILCs appear as high inertia so as to avoid AC bus high-frequency oscillation during loading transients. It's noteworthy that suitable virtual damp could availably suppress active power and frequency fluctuation and regulate $\mathrm{AC}$-side frequency extremely smoothly.

This paper is organized as follows. Section II overviews droop control methods of DGs within DC and AC subgrids. Section III explains the improved ILCs control scheme including proportional power sharing and application of synchronverter technology. Simulation results are given in Section IV and experimental results are presented in Section $\mathrm{V}$ with conclusions in Section VI.

\section{Autonomous Control Within Subgrids}

\section{A. Droop Control of DGs within AC Subgrid}

DGs linking to the AC bus have widely adopt droop control scheme [12]. As shown in Fig. 2, active and reactive power within AC subsystem are relevant to the references of voltage frequency and amplitude, respectively. The distributed sources can automatically share the total active power and reactive power demand in proportion to their rated power and maitain the stable operation of the AC subgrid.The droop equations for obtaining voltage frequency and amplitude of $x$ th DG unit are presented as in

$$
f_{\mathrm{ac} \_\mathrm{H}}=f_{\mathrm{x}}^{\prime}+m_{\mathrm{x}} P_{\mathrm{a}, \mathrm{x}} ; U_{\mathrm{ac} \_\mathrm{H}}=U_{\mathrm{ac}, \mathrm{x}}^{\prime}+n_{\mathrm{x}} Q_{\mathrm{a}, \mathrm{x}}
$$

where $f_{\mathrm{ac} \_\mathrm{H}}$ and $U_{\mathrm{ac} \_\mathrm{H}}$ are the maximum allowable voltage frequency and amplitude respectively; $m_{x}$ and $n_{x}$ are the droop coefficients. (a) P-f Droop

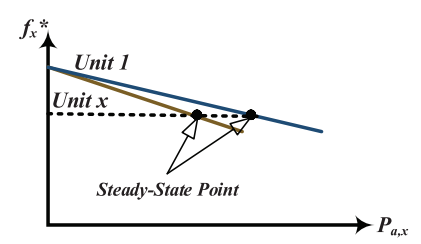

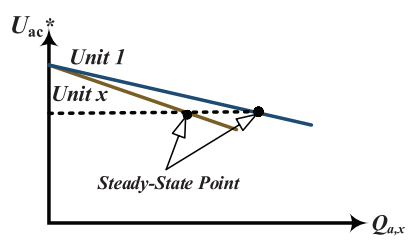

(b) $Q-U_{\text {ac }}$ Droop
Fig. 2. $P-f$ and $Q-U_{\text {ac }}$ droop characteristic in AC subgrid.

Upon reaching steady state, these parallel DGs come to be one prevailing frequency autonomously as presented by the single dashed horizontal line. Similarly, we will also get one voltage amplitude ignoring line impedance at the steady working point. Consequently, the AC subsystem will nicely meet such $P-f$ and $Q-U_{\text {ac }}$ droop characteristics as follows

$$
\begin{gathered}
\left\{\begin{array}{l}
f_{\mathrm{ac} \_\mathrm{H}}=f_{\mathrm{ac}}^{\prime}+m_{\mathrm{ac}} P_{\mathrm{ac}} \\
U_{\mathrm{ac} \_\mathrm{H}}=U_{\mathrm{ac}}^{\prime}+n_{\mathrm{ac}} Q_{\mathrm{ac}}
\end{array}\right. \\
\left\{\begin{array}{l}
m_{\mathrm{ac}}=\left(f_{\mathrm{ac} \_\mathrm{H}}-f_{\mathrm{ac} \_\mathrm{L}}\right) / P_{\mathrm{ac} \_\max } \\
n_{\mathrm{ac}}=\left(U_{\mathrm{ac} \_\mathrm{H}}-U_{\mathrm{ac} \_\mathrm{L}}\right) / Q_{\mathrm{ac} \_\max }
\end{array}\right.
\end{gathered}
$$

where $m_{\mathrm{ac}}$ and $n_{\mathrm{ac}}$ are the combined droop coefficients of the AC bus; $f_{\text {ac } L}$ and $U_{\text {ac } L}$ are the minimum allowable voltage frequency and amplitude respectively; $P_{\mathrm{ac} \_ \text {max }}$ and $Q_{\mathrm{ac} \_ \text {max }}$ are the rated active and reactive power capacity of AC subgrid respectively.

\section{B. Droop Control of DGs within DC Subgrid}

Comparing to $\mathrm{AC}$ subgrid, the $\mathrm{DC}$ subgrid is a little easier without considering reactive power, frequency, and phase issues. As shown in Fig. 3, DC bus voltage will fall down linearly with the output active power increasing [13]. Without taking line impedance into consideration, DGs remain the same voltage in the steady-state point. The droop equation of $y$ th DG connected to the DC bus is given by

$$
U_{\mathrm{dc} \_\mathrm{H}}=U_{\mathrm{dc}, \mathrm{y}}^{\prime}+k_{\mathrm{y}} P_{\mathrm{dc}, \mathrm{y}}
$$

where $U_{\mathrm{dc} \text { H }}$ is the maximum output voltage at no load condition, $k_{\mathrm{y}}$ is the droop coefficient of the $y$ th DG unit. Similarly, mathematic model of the total DC subsystem can be expressed as follows

$$
\begin{gathered}
U_{\mathrm{dc} \_\mathrm{H}}=U_{\mathrm{dc}}^{\prime}+k_{\mathrm{dc}} P_{\mathrm{dc}} \\
k_{\mathrm{dc}}=\left(U_{\mathrm{dc} \_\mathrm{H}}-U_{\mathrm{dc} \_}\right) / P_{\mathrm{dc} \_\max }
\end{gathered}
$$

where $k_{\mathrm{dc}}$ is the DC bus droop coefficient and $P_{\mathrm{dc} \text { max }}$ is the maximum output active power of the DC subgrid.

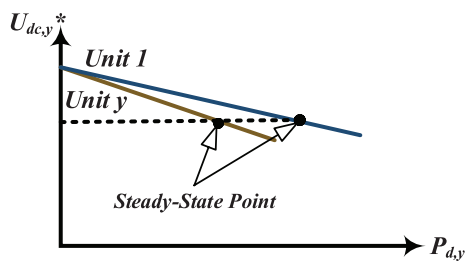

Fig. 3. $P-U_{\mathrm{dc}}$ droop characteristic in DC subgrid.

\section{Improved ILCs Control Strategy}

ILCs are aimed to properly manage the power flows between AC and DC subgrids such that proportional power sharing throughout hybrid microgrids is guaranteed. Besides, due to inertia-less feature of converters, microgrids with high penetration rate of inverter-interfaced DGs usually suffer 


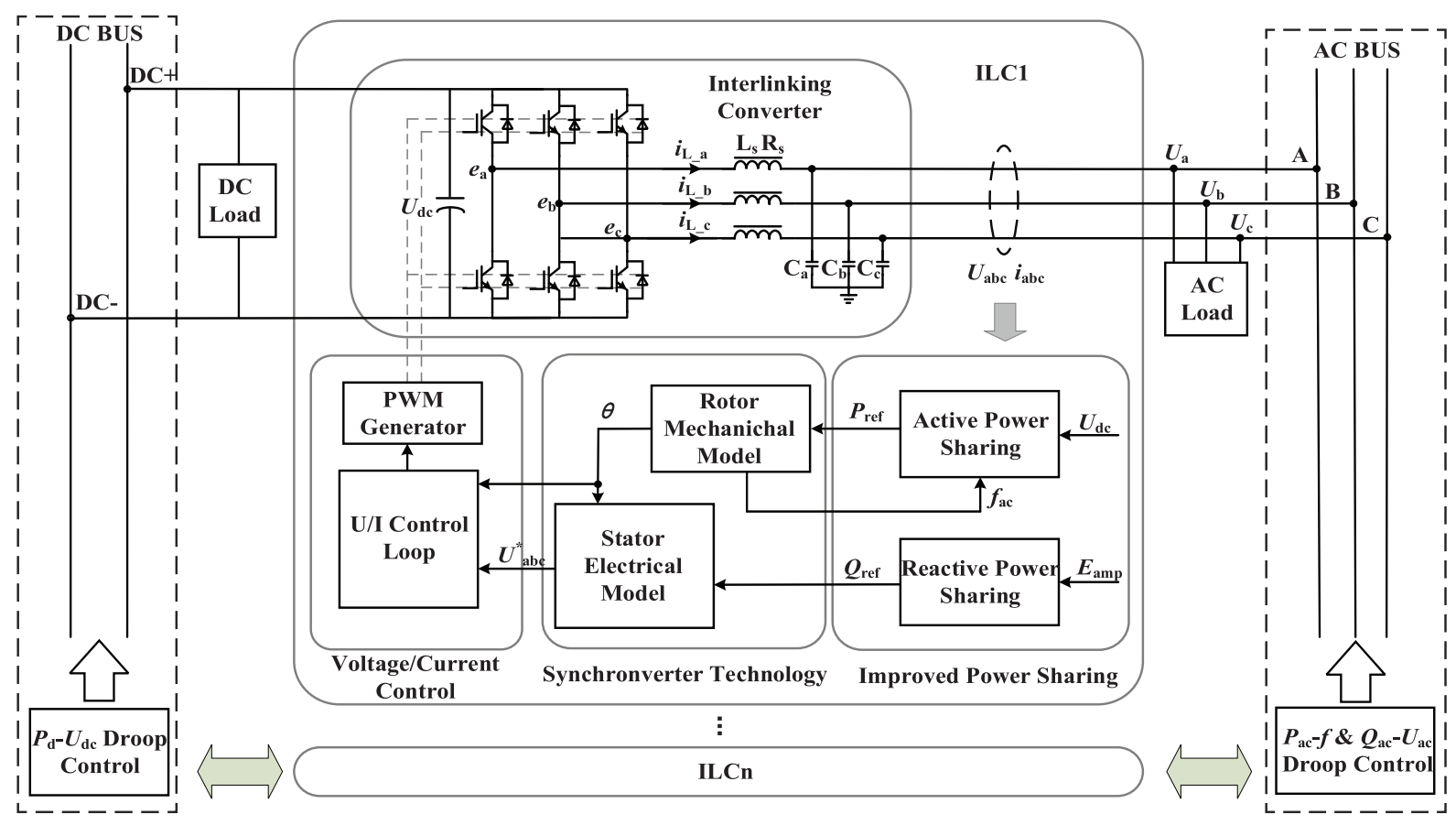

Fig. 4. Control block diagram of hybrid AC/DC microgrids.

from heavy power issues such as poor dynamic frequency stability. And large frequency variation again aggravates this matter. To solve this problem, ILCs should be also responsible for the regulation of AC subgrid frequency stability instead of only depending on the DGs inside of the AC subsystem. What's more, it's also considerable for ILCs to provide uninterruptable power supply for AC-side extreme loads during AC subgrid faults events in an islanded mode.

As a consequence, the improved ILCs control strategy is proposed to exchange proportional power flows, improve the $\mathrm{AC}$-side frequency dynamic stability, and offer voltage support to the AC subsystem during faults events. Based on such control objectives, the ILC is connected to the AC bus via a LC filter so that it can be served as a voltage-controlled source with proper control method and offer voltage support to the AC subgrid shown in Fig. 4. The ILCs control strategy adopts direct frequency control to ensure proportional active power sharing and makes the microgrid appear as high inertia and damp with the application of synchronverter technology. The complex voltage command $U_{\text {abc }}^{*}$ is tracked by a traditional double PI voltage and current control of an inverter. The double control loop is well known, and hence no further clarification here.

\section{A. Proportional Power Sharing Control}

Rational power sharing is the main task for ILCs in the island mode, which includes active power and reactive power [2]. AC-side frequency is a global parameter and is the same throughout AC subgrid. On the basis of (2), any increase in power demand in the AC subsystem will follow by a decrease in $\mathrm{AC}$ bus frequency. Therefore, the relative amount of frequency variation range is anavailable reflection of the relative output active power of the AC subgrid. Similarly, the relative amount of DC subgrid voltage variation is a reliable indication of the relative active power of DC subsystem. However, AC frequency and DC terminal voltage are two different types of droop variables corresponding to $\mathrm{AC}$-side and DC-side active power. To address this problem, these variations can be brought to a common per unit range by [6]

$$
\left\{\begin{array}{l}
f_{p u}=\frac{f_{\mathrm{ac}}-0.5\left(f_{\mathrm{ac} \_\mathrm{H}}+f_{\mathrm{ac} \_\mathrm{L}}\right)}{0.5\left(f_{\mathrm{ac} \_\mathrm{H}}-f_{\mathrm{ac} \_\mathrm{L}}\right)} \\
U_{d c, p u}=\frac{U_{\mathrm{dc}}-0.5\left(U_{\mathrm{dc} \_\mathrm{H}}+U_{\mathrm{dc} \_\mathrm{L}}\right)}{0.5\left(U_{\mathrm{dc} \_\mathrm{H}}-U_{\mathrm{dc} \_\mathrm{L}}\right)}
\end{array}\right.
$$

Setting the per-unit frequency and per-unit DC voltage equal in the hybrid microgrid, the total active power demanded by both $\mathrm{AC}$ and DC subgrids are shared among all DGs proportional to their rated power. Traditional methods employed in ILCs use the error of the normalized variations $\left(f_{p u}-U_{d c, p u}\right)$ to calculate the reference active power and usually realized by current-controlled method [6-8]. Such control methods only ensure rating active power distribution but can't offer voltage support to AC bus and even deteriorate the AC subgrid voltage quality to a degree. As a consequence, this scheme adopts direct frequency control method to improve frequency response meanwhile maintaining the function of conventional control scheme. According to (2) (3) (5) (6) and setting $\mathrm{AC}$ and $\mathrm{DC}$ instantaneous active power to be equal, real-time dynamic reference frequency is obtained as

$$
f_{\text {ref }}=f_{\mathrm{ac} \_\mathrm{H}}-\frac{\left(f_{\mathrm{ac} \_\mathrm{H}}-f_{\mathrm{ac} \mathrm{L}}\right) P_{\mathrm{dc} \_\max }}{\left(U_{\mathrm{dc} \_\mathrm{H}}-U_{\mathrm{dc} \_\mathrm{L}}\right) P_{\mathrm{ac} \_ \text {max }}}\left(U_{\mathrm{dc} \_\mathrm{H}}-U_{\mathrm{dc}}^{\prime}\right)
$$


As long as controlling instantaneous AC-side frequency $f_{\text {ac }}$ and reference frequency $f_{\text {ref }}$ to be equal, the output active power of AC and DC subgrids will naturally be proportional to each rated power upon reaching steady state. According to (8) and droop control within subgrids, ILC droop characteristic can be described as Fig. 5, where $e_{\mathrm{f}}$ is the error of reference frequency $f_{\text {ref }}$ and feedback value $f_{\text {ac }}$.

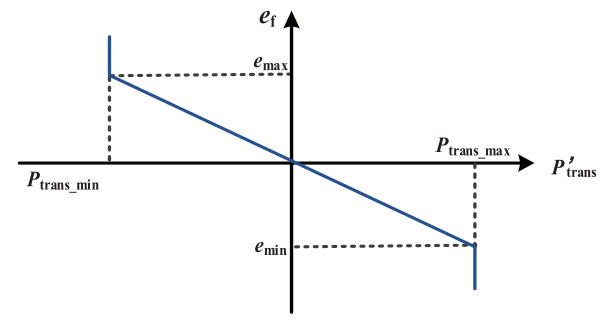

Fig. 5. $P-f$ droop characteristic of ILCs.

Specific direct frequency control method is shown in Fig. 6 , DC-side voltage is measured and plugged into (8) to get real-time reference frequency corresponding to the DC-side voltage variation. Then $f_{\text {ref }}$ is sent to a low-pass filter (LPF) so as to avoid high frequency harmonics resulting in the fluctuation of frequency and active power. $K_{\mathrm{f}}$ is the droop coefficient of ILCs used to calculate exchanged active power according to Fig. 5. Integration element $1 / J_{\mathrm{fS}}$ is applied to realize non-error adjustment regulation for frequency corresponding to power sharing and increase frequency inertia interrelated to the virtual damp of synchronverter meanwhile. By controlling AC-side frequency to regulate transferred active power, it can not only manage proportional power sharing between the dual subgrids but also improve dynamic frequency stability to a degree with the application of frequency integration element. As for negative transferred power control part shown in Fig. 6, the ILC is regarded as a distributed source connected to the AC bus and adopt a $Q-U_{\text {ac }}$ droop control so as to enable the DC subgrid share proportional reactive power with the $\mathrm{AC}$ subsystem.

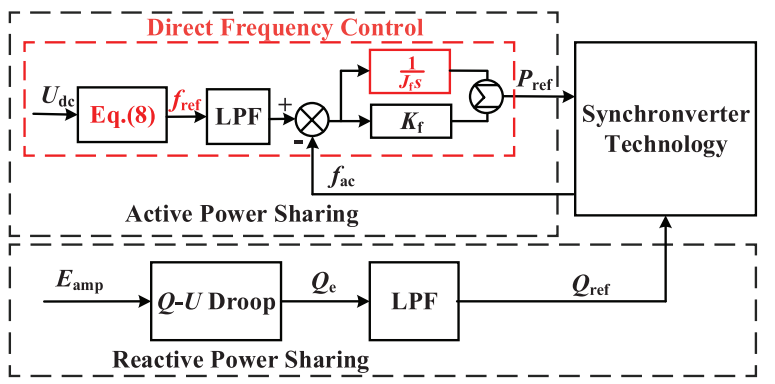

Fig. 6. Power sharing control block.

\section{B. Application of Synchronverter Technology in ILCs}

In order to enable ILC appear as high inertia and damp to enhance the dynamic frequency stability during AC-side load transients, this part applies the synchronverter technology to the ILC control scheme. The DC subgrid contains of various
DGs with storage elements shown in Fig. 1. And thus there's no extra energy storages demanded for ILCs.

Synchronverter is to apply the mathematic model of synchronous generator into converters to mimic good characteristics of a synchronous generator [14]. The simplified mathematic model of synchronous generator can be summarized by rotor mechanical model and stator electrical model shown respectively as [15]

$$
\begin{aligned}
& \left\{\begin{array}{l}
J \dot{\omega}=T_{m}-T_{e}+D_{p}\left(\omega_{n}-\omega\right) \\
T e=M_{f} i_{f}\left\langle i_{L}, \widetilde{\sin \theta}\right\rangle
\end{array}\right. \\
& \left\{\begin{array}{l}
e=\omega M_{f} i_{f} \widetilde{\sin \theta} \\
Q=-\omega M_{f} i_{f}\left\langle i_{L}, \widetilde{\cos \theta}\right\rangle
\end{array}\right.
\end{aligned}
$$

where $J$ and $D_{\mathrm{p}}$ are imaginary inertia and damp of all the parts rotating with the rotor. $\omega, T_{\mathrm{m}}, T_{\mathrm{e}}, e$, and $Q$ are the respectively synchronverter angular frequency, virtual mechanical torque, the electromagnetic torture, the converter potential voltage, and the reactive power of the synchronverter respectively. $I_{\mathrm{f}}$ is the field-excitation current and $M_{\mathrm{f}}$ is the maximum mutual inductance between the stator windings and the field winding. $i_{\mathrm{L}}$ is the output inductor current. Denote

$$
\widetilde{\cos \theta}=\left[\begin{array}{c}
\cos \theta \\
\cos (\theta-2 \pi / 3) \\
\cos (\theta+2 \pi / 3)
\end{array}\right], \widetilde{\sin \theta}=\left[\begin{array}{c}
\sin \theta \\
\sin (\theta-2 \pi / 3) \\
\sin (\theta+2 \pi / 3)
\end{array}\right] .
$$

The synchronverter technology control part is shown in Fig. 7. Reference active and inactive power are all calculated by the power sharing partshown in Fig. 6 and they can be regulated separately by synchronverter relying on the mathematic model presented in (9) and (10).

In the mechanical control part, $J$ and $D_{p}$ represent the associated ILC's virtual inertia and damp coefficients, respectively. According to (9), dynmics of the system frequency $\omega$ can be optimized and appear as high inertia by using proper inertia coefficient. It does help to ease the problem of severe frequency oscillations during AC-side load transients. Damp factor $D_{\mathrm{p}}$ can suppress the oscillation of active power [16]. It is a key factor to completely damp frequency fluctuation making it possible to regulate AC-side frequency extremely smoothly, which is not available just relying on virtual inertia. During the process of damping coefficient debugging, there's still once oscillation in frequency and active power while $D_{\mathrm{p}}$ is too small during transient regulation process. Only until virtual damp coefficient is large enough can the AC-side frequency regulate absolutely smoothly without any fluctuation. And in this schemeit can be also used to increase system inertia combined with the inertia element $J_{\mathrm{f}}$ in Fig. 6. In islanded hybrid microgrid, frequency of $\mathrm{AC}$ subgrid will change with the load changing because of $P-f$ droop charateristic while $\omega_{\mathrm{n}}$ is one constant value. Once upon the 
steady-state point, the damping power $P_{\text {damp }}=\omega D_{\mathrm{p}}\left(\omega_{\mathrm{n}}-\omega\right)$ is not equal to zero according to (9). Then the steady-state output active power $P_{\mathrm{e}}$ of interlinking converter is the sum of damping power $P_{\mathrm{damp}}$ and $P_{\mathrm{m}}$. As a consequence, $1 /\left(J_{\mathrm{fs}}\right)$ is used to be the intergal part of a PI controller employed for forcing the steady-state difference between AC and DC subsystem output active power to be zero in order to ensure accurate power sharing. On the other hand, during the process of dynamic regulation, $1 /\left(J_{\mathrm{fs}}\right)$ can be served as an inertia element with time delay to increase system inertia. What's more, with the application of synchronverter technology, AC-side frequency can be got from system angular speed instead of using a voltage phase-locked loop (PLL) like the traditional ILCs droop control scheme.

In the electrical control part shown in Fig. 7, the regulation of reactive power $Q$ flowing out of ILCs can be realized similarly. The difference between the reference value $Q_{\text {ref }}$ and the reactive power $Q$ is tracked and fed into an integrator with a gain $1 / \mathrm{K}$ togenerate $M_{\mathrm{f}} i_{\mathrm{f}}$, which will be put into (10) to get the final reference voltage and realize the power control by the voltage/current double closed control with zero steady voltage error meanwhile offering support to the $\mathrm{AC}$ bus.

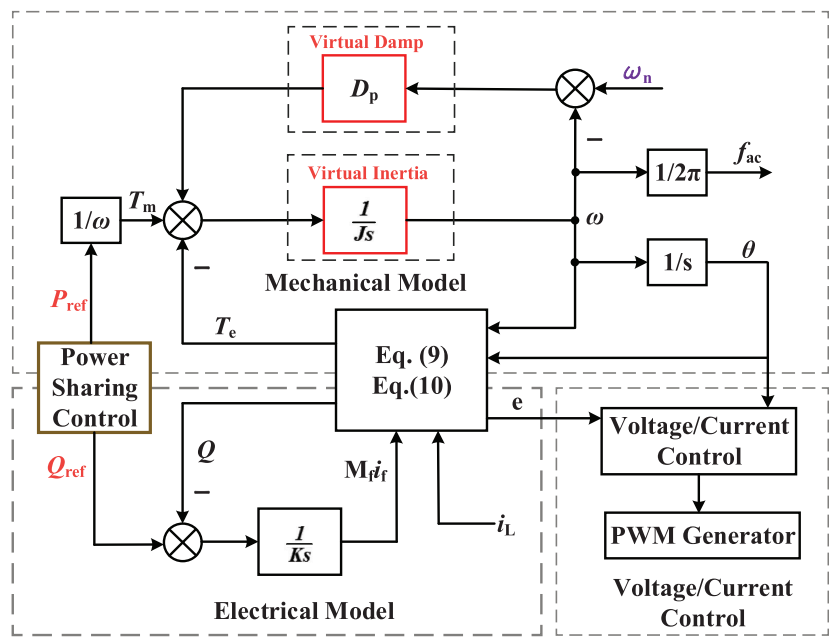

Fig. 7. Application of synchronverter control block.

\section{Simulation Results}

In this section, time-domain simulation is used to verify the analytical studies presented in the previous sections. The simulations have been carried out by using Matlab/Simulink. The tested structure of hybrid microgrid comprising a DC subsystem and an AC subsystem is shown in Fig. 8. The ILC adopts traditional six-switch DC/AC converter presented in Fig. 4 and links to the AC bus through an LC filter. Integrated by various DGs, the AC subsystem is modeled by an inverter controlled by $P / f$ and $Q / U_{\text {ac }}$ droop control methods described in Fig. 2. Due to the wide distribution of DGs in the actual microgrid, the line impedance between PCC and DGs should be also considered. As for the DC subsystem, it's modeled by one voltage-controlled source with $P / U_{\mathrm{dc}}$ droop characteristic.

System parameters of the tested hybrid microgrid are given in Table I. The total rating power of AC subgrid is $10 \mathrm{~kW}$ over an allowable frequency variation of $49 \mathrm{~Hz}<f<51 \mathrm{~Hz}$ and an $\mathrm{AC}$ voltage range $215 \mathrm{~V}<U_{\mathrm{ac}}<225 \mathrm{~V}$. The corresponding value of the DC subsystem is $10 \mathrm{~kW}$ over an available DC voltage range $590 \mathrm{~V}<U_{\mathrm{dc}}<600 \mathrm{~V}$. To verify the feasibility of this proposed control strategy, loads connected to the two subgrids are switched between "light" and "heavy" condition and the test is organized as two transient cases. At the beginning, the hybrid microgrid works in a steady state under light load condition. At 1s, DC-side load switches from light to heavy. At 3s, when the microgrid reachessteady state, ACside load changes from the light condition to a heavy mode. Waveforms of frequency and power are presented as follows with analysis.

TABLE I

System Parameters

\begin{tabular}{l|l}
\hline \hline Parameter & Value \\
\hline DC Subgrid Rated Power & $P_{\text {ac,max }}=10 \mathrm{~kW}$ \\
AC Subgrid Rated Power & $P_{\mathrm{dc}, \max }=10 \mathrm{~kW}$ \\
DC Voltage Range & $590 \mathrm{~V}<U_{\mathrm{dc}}<600 \mathrm{~V}$ \\
AC Voltage Range & $215 \mathrm{~V}<U_{\mathrm{ac}}<225 \mathrm{~V}$ \\
AC Frequency Variation & $49 \mathrm{~Hz}<\mathrm{f}<51 \mathrm{~Hz}$ \\
\hline Circuit Parameter & Value \\
\hline ILC DC-side Capacity & $C_{\mathrm{dc}}=4700 \mathrm{uF}$ \\
LC Filter & $L_{\mathrm{f}}=3 \mathrm{mH} C_{\mathrm{f}}=35 \mathrm{uF}$ \\
Line Impedance & $L_{\mathrm{Z}}=2 \mathrm{mH} R_{\mathrm{z}}=0.2 \Omega$ \\
\hline Contol Parameter & Value \\
\hline Inertia Coefficient & $J_{\mathrm{f}}=2 \mathrm{e}-5 J=0.06$ \\
Damp Coefficient & $D_{\mathrm{p}}=50$ \\
\hline Load Parameter & Value \\
\hline DC-side Load & $1.3 \mathrm{~kW}$ (Light) $6.3 \mathrm{~kW}$ (Heavy) \\
AC-side Load1 & $3.7 \mathrm{~kW}$ (Light) $8.7 \mathrm{~kW}$ (Heavy) \\
AC-side Load2 (Faults) & $3.7 \mathrm{~kW}$ (Light) $6.5 \mathrm{~kW}$ (Heavy) \\
\hline \hline
\end{tabular}

\section{A. Improved Frequency Transient Stability}

This paper is devoted to the improvement of frequency dynamic stability during AC-side load transients. Fig. 9 describes the waveforms of frequency adopting different ILCs control methods under the same condition. At 1s, DCside loads switch from the light mode to heavy mode and AC subgrid autonomously outputs more power to share the power supply with frequency decrease. While AC-side loads increase a lot suddenly at $3 \mathrm{~s}$, the $\mathrm{AC}$ bus frequency controlled by the traditional ILCs control strategy in Ref [6] could oscillateseverely and even over modulated beyond the normal frequency operating range $(49 \mathrm{~Hz} \sim 51 \mathrm{~Hz})$ as shown in Fig. 8(a), which has a terrible frequency transient stability obviously. As for the simulation results of this improved strategy presented in Fig. 9(b), AC-side frequency could regulate smoothly without any oscillation from the current point to the steady-state value no matter what DC-side or AC-side load changes suddenly. Consequently, the simulation results 


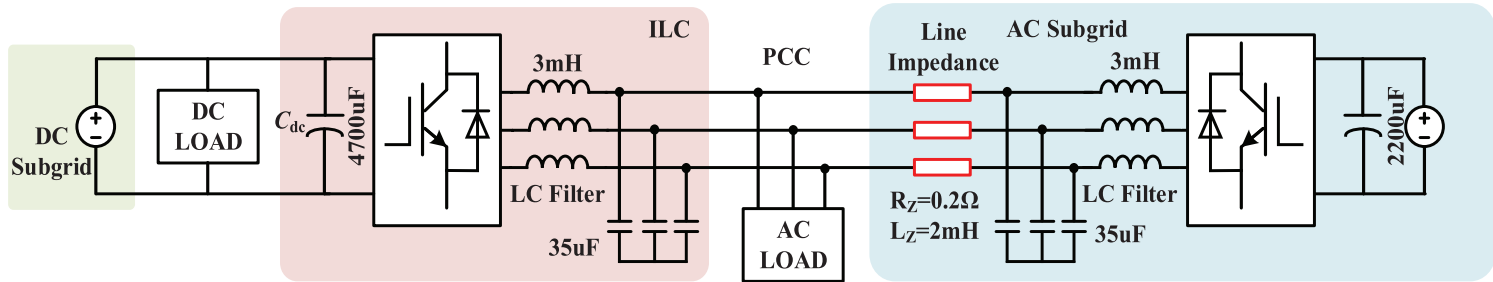

Fig. 8. Structure of the tested hybrid AC/DC microgrid.

in Fig. 9 prove that this improved strategy is effective and has a better performance on the frequency transient stability.

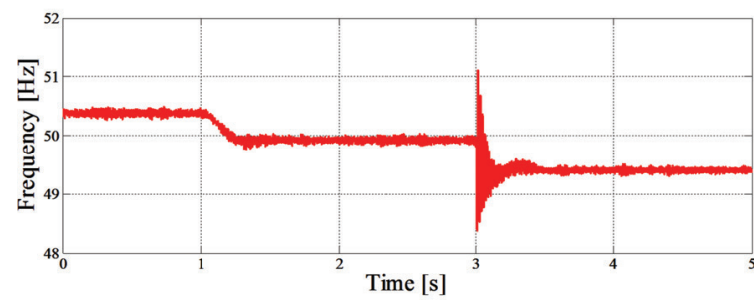

(a) traditionalcontrol scheme

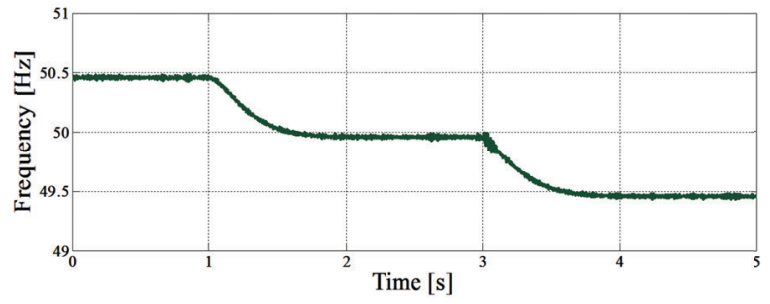

(b) improved control scheme

Fig. 9. Simulation waveforms of AC-side frequency for load transitions.

It's worthwhile to note that virtual damp plays an important role in the dynamic response of frequency corresponding to the AC-side active power. Suitable damp coefficient can completely damp the AC-side frequency and active power-

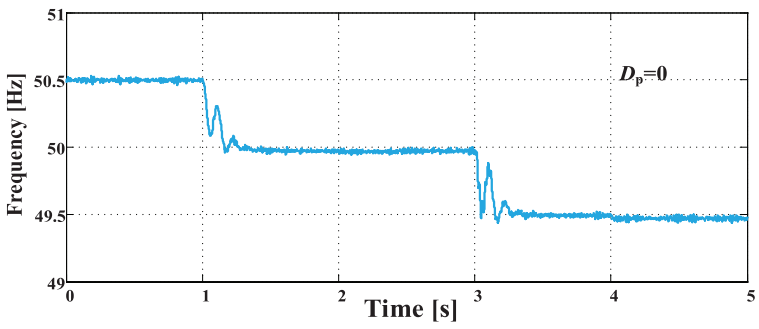

(a) without damp

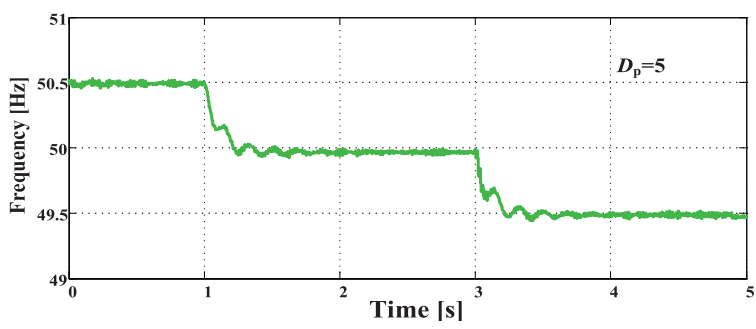

(b) small damp

Fig. 10. Simulation waveforms of AC-side frequency with different damp. fluctuation and makes it possible to regulate smoothly during load transients. Fig. 10 gives the test results of AC-side frequency with different virtual damp coefficients. There are obvious frequency fluctuations during either DC-side or AC-side load transient without the application of virtual damp $\left(D_{\mathrm{p}}=0\right)$. After given a small damp coefficient $\left(D_{\mathrm{p}}=5\right)$, fluctuations of frequency are suppressed availably although there're still some slight oscillations. While the virtual damp is large enough $\left(D_{\mathrm{p}}=50\right)$, the frequency can regulate absolutely smoothly shown in Fig. 9(b).

\section{B. Proportional Power Sharing}

Besides dynamic frequency stability, proportional power sharing is the main task of ILCs that must be achieved. To facilitate the results analysis, set DC and AC subgrid rated power to be the same that both are $10 \mathrm{~kW}$ as presented in Table I. Therefore, as long as the two subgrids output power can be equal under different load conditions in the steady state, then it will demonstrate that the two subgrids output power are proportional to their rated energy value. From Fig. 11 , in the first steady state from $0 \mathrm{~s}$ to $1 \mathrm{~s}$, the DC-subgrid output power is about $2.5 \mathrm{~kW}$ equal to the $\mathrm{AC}$-side output value. In the second steady state from $1 \mathrm{~s}$ to $3 \mathrm{~s}$, the output active power of DC subsystem is $5 \mathrm{~kW}$ similar to the AC subgrid; lastly in the third steady state, the two subgrids output powers are still approximately equal to each other. As a result, this improved control scheme can achieve the basic control objective about proportional power sharing.

As for the dynamic power response of both $\mathrm{AC}$ and $\mathrm{DC}$ subsystem, it's because of the DC-side load transient at $1 \mathrm{~s}$ that DC subgrid outputs large amount of power at once but does few effects on the other side subgrid of ILCs. However, with the application of virtual inertia and virtual damp, the AC subsystem can still output power smoothly at $3 \mathrm{~s}$ instead of changing largely or even overshooting just like the waveform of DC subsystem at 1s despite of AC-side load transient. What's more, at 1s due to the DC-side load changes the output power of DC subgrid increases a lot at once to meet the power demand. In order to balance the two subsystem power supply, ILCs manage power flows from AC-side to the DC-side. As Fig. 11(c) shows that the value of ILCs output power is negative. Similarly, from $3 \mathrm{~s}$ to $5 \mathrm{~s}$ AC-side load is heavier than the DC subgrid, the ILC exchange power from DC subsystem to AC subsystem and value of the transferred power is positive shown in Fig. 11(c).

Dynamic responses of $\mathrm{AC}$ current under the two transient events are given in Fig. 11(d) and (e). Either increasing 


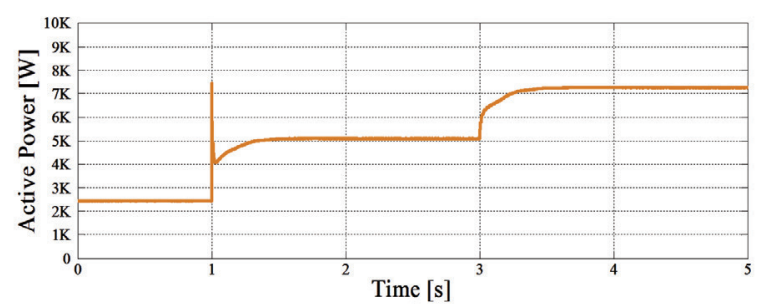

(a) DC subgrid output active power

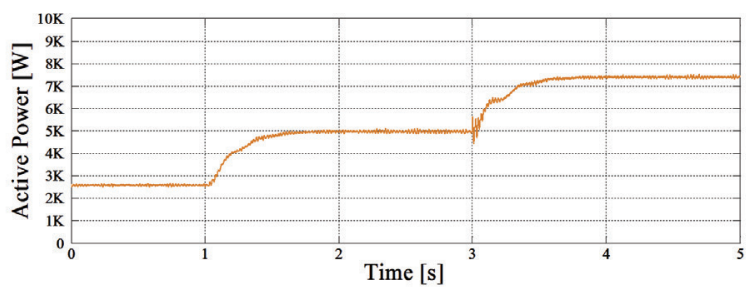

(b) AC subgrid output active power

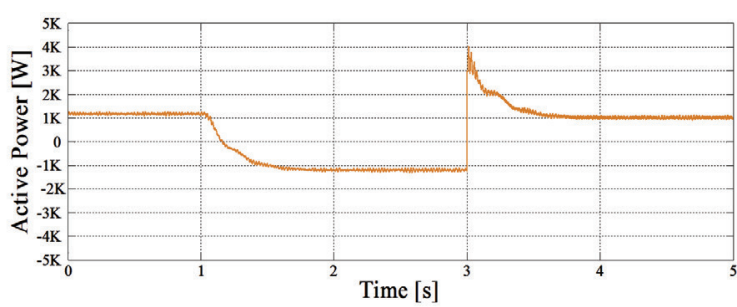

(c) ILC transferred active power

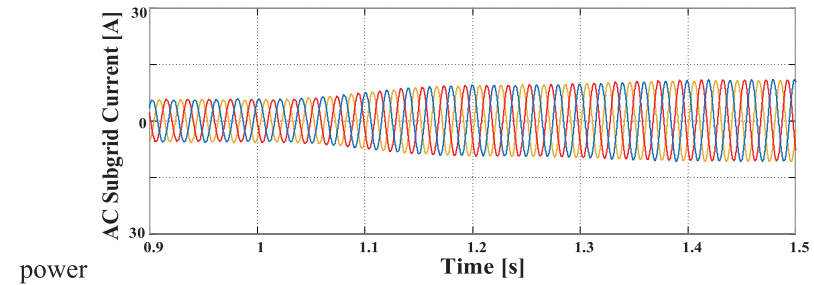

(d) transferred active power

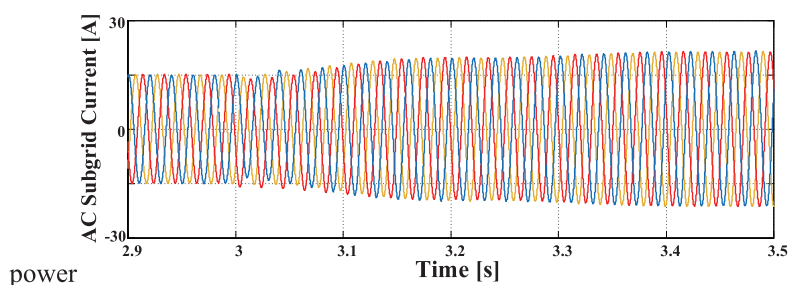

(e) DC subgrid output active power

Fig. 11. Simulation waveforms of DC-AC subgrid and ILC output Active power and $\mathrm{AC}$ currents for load transitions.

DC-side load or AC-side load, AC subsystem outputs more current to realize proper power sharing and meet all the load demand. During the dynamic regulation progress, the peak currents change smoothly maintaining a stable operation of hybrid microgrid.

\section{Higher Reliability Test During AC Subgrid Faults Events in Islanded Mode}

In order to further validate the higher reliability of this scheme, in this section the paper simulates a subgrid fault event in islanded mode by breaking down the connection between AC-side loads and AC bus. As Fig. 12 shows, at the beginning the hybrid microgrid works in a steady operation; then at $1 \mathrm{~s}$, the $\mathrm{AC}$ bus disconnects with the loads all of a sudden, it's obvious that AC-subsystem output power changes to be zero later and ILCs manage power from DC bus to the AC-side loads to provide uninterrupted power supply. As for the frequency, it can change stably and reach steady state during the process of transient regulation. Besides, in

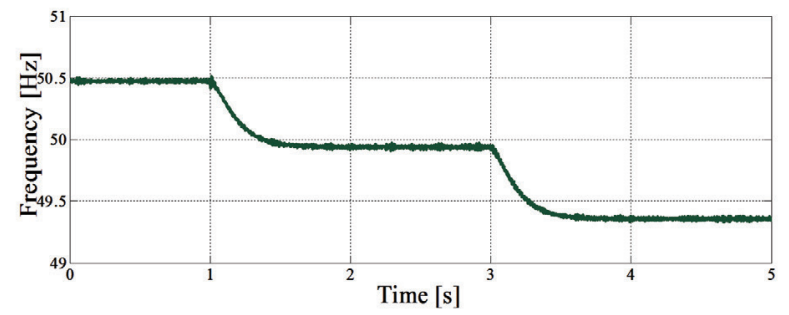

(a) AC-side frequency

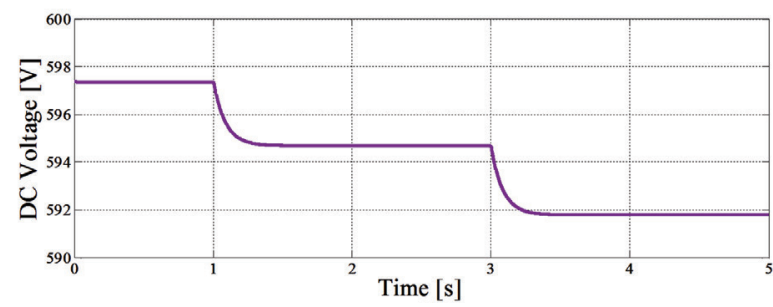

(b) DC-side voltage

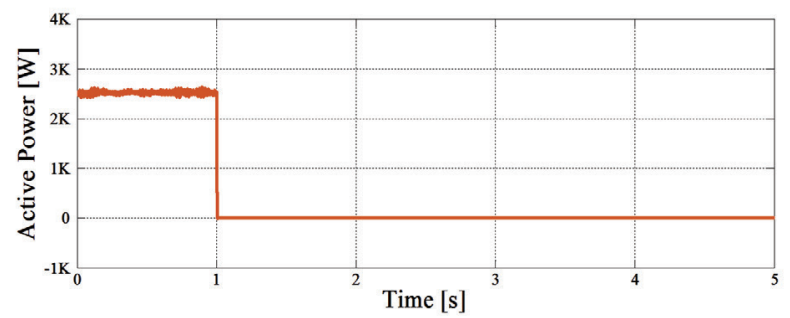

(c) AC subgrid output active power

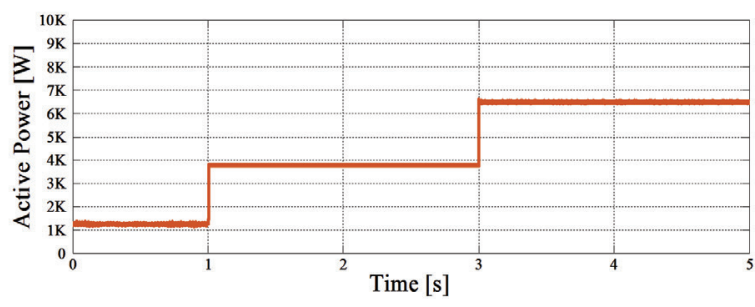

(d) transferred active power

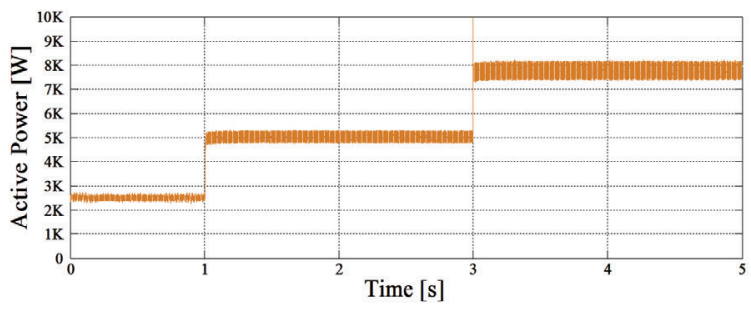

(e) DC subgrid output active power

Fig. 12. Simulation waveforms of DC-AC subgrid and ILC output active power for load transitions. 
order to further test the dynamic frequency stability, the ACside loads change from the light to heavy mode at $3 \mathrm{~s}$. As we can see from Fig. 12, AC-side subgrid frequency can still regulate smoothly within allowable variation without the assistance of $\mathrm{AC}$ bus in the islanded operation. Compared to the general normalized droop control, this enhanced method performs higher reliability and dynamic frequency stability.

\section{EXPERIMENTAL RESULTS}

The proposed control scheme was further vilified experimentally by the OPAL-RT real-time digital simulator. The proposed control scheme was further vilified experimentally by the OPAL-RT real-time digital simulator. Experimental system parameters and the tested system structure are the same as those used in the simulation and presented in Table $\mathrm{I}$ and Fig. 8. A standard DC/AC inverter controlled by $P / f$ and $Q / U_{\text {ac }}$ droop control methods and a normal line impedance $(\mathrm{R}=0.2 \Omega, \mathrm{L}=2 \mathrm{mH})$ are formed to be the AC subgrid in the tested hybrid microgrid. DC subgrid is realized by a voltage-controlled DC source with droop characteristic. The maximum output active power of both $\mathrm{AC}$ and DC subsystem is set to be $10 \mathrm{~kW}$ for the sake of discussion. With respect to the application of synchronverter technology, the ILC adopts a standard DC/AC converter with LC filter to be controlled as a voltage-controlled source with high inertia. This scheme is devoted to deal with the weakness of poor dynamic frequency stability in traditional normalized droop control methods. Therefore, only active power flows and frequency performance are required to be observed in this test. For simplicity, there're two converters controlled with droop characteristics shown in the second section to represent AC and DC subgrid separately. And the other two converters were paralleled and connected to the $\mathrm{DC}$ and $\mathrm{AC}$ buses to serve as ILCs. The experiments were performed under the condition of $10 \mathrm{kHz}$ switching frequency and carried out as following conditions.

The first test is shown in Fig. 13 with the two subgrids both working in normal state with a DC load of $1.3 \mathrm{~kW}$ and an AC load of $3.7 \mathrm{~kW}$. At the beginning, AC-side loads demanded more power than loads of DC subgrid, and thus the ILCs exchange about $1.2 \mathrm{~kW}$ power from the DC subsystem to AC subsystem to share the output power proportionally shown in Fig. 13(b). At 2s, DC-side load is increased to be $6.3 \mathrm{~kW}$ leading to more supplied active power of DC subsystem than AC subsystem. While reaching to the steady state, the output active powers of $\mathrm{AC}$ and $\mathrm{DC}$ subgrid both equal to be $5 \mathrm{~kW}$ by exchanging $-1.3 \mathrm{~kW}$ power. At $6 \mathrm{~s}$, the ACside load increases by $5 \mathrm{~kW}$ and the exchanged active power of ILCs changed from $-1.3 \mathrm{~kW}$ to $1.2 \mathrm{~kW}$. In the whole experiment, the output active power of DC Subgrid and AC Subgrid can be always equal on steady state as presented in Fig. 13(b), which proves that this proposed scheme can manage proportional power sharing between the dual subgrids. Fig. 13(a) shows that AC-side frequency appears as high inertia and damp and performs good dynamic regulation ability, which can always change smoothly without any os-

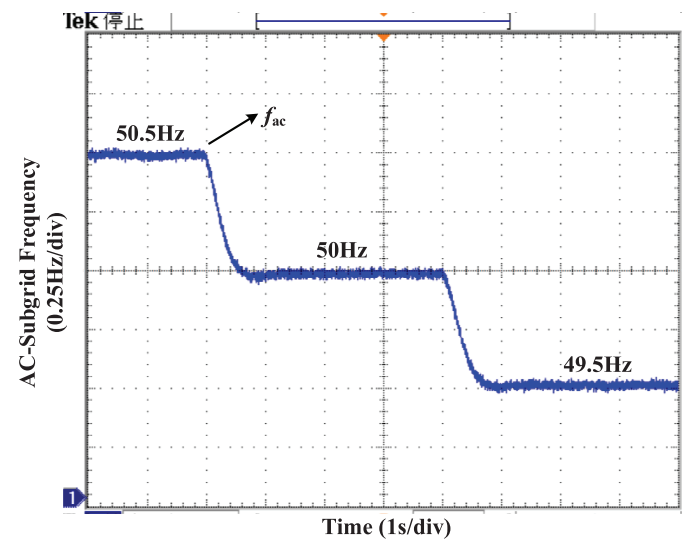

(a) AC-side frequency

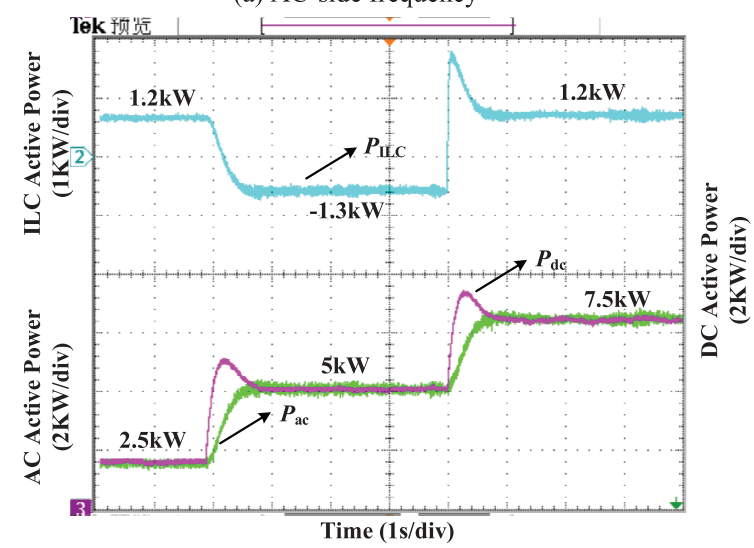

(b) Active power

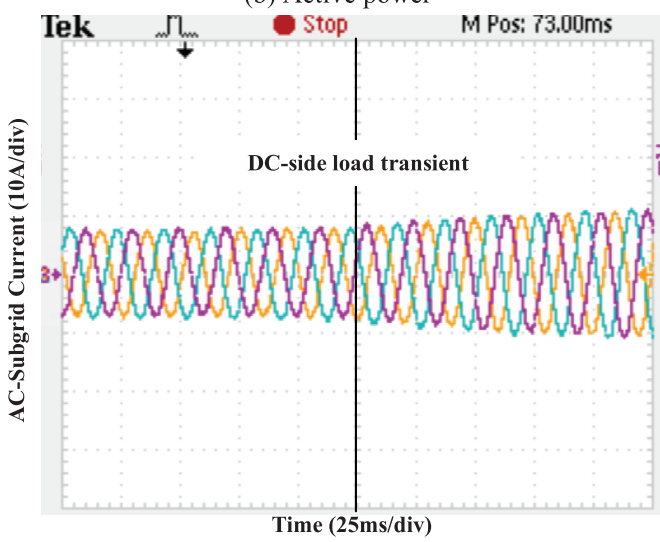

(c) AC current during DC-side load transient

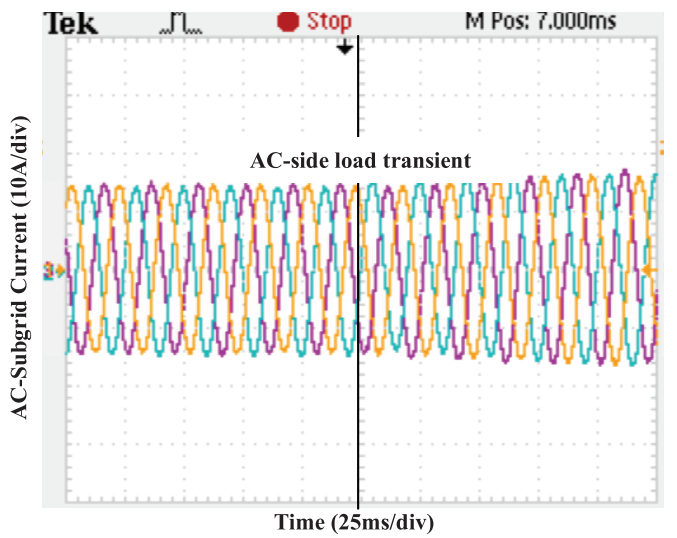

(d) AC current during AC-side load transient

Fig. 13. Waveforms of hybrid microgrid under normal condition. 
cillation during loads transients. Besides, dynamic responses of the AC subsystem under the two transient conditions are presented in Fig. 13(c) and (d). The AC current can also regulate smoothly without distortion during the dynamic regulation progress ensuring the safe and feasible operation the $\mathrm{AC}$ subgrid.

Experimental results of the other test is shown in Fig. 14 with AC subgrid faults while operating in islanded mode. Similarly, in the first two seconds the hybrid AC/DC microgrid operated steadily in a normal state. At $2 \mathrm{~s}$, disconnect the inverter served as AC subgrid from AC-side loads to equal the state of AC subsystem faults events. Increase AC-side loads by $2.8 \mathrm{~kW}$ suddenly at $6 \mathrm{~s}$ to make further verification of dynamic frequency stability. Fig. 14(a) shows that ACside frequency can always change smoothly without any fluctuation and verifies that this improved method optimize the frequency transient stability to a great extent. Fig. 14(b) shows that DC subgrid and ILCs could still operate steadily and provide uninterrupted power supply for the AC-side extreme loads without AC subsystem. What's more, the whole experiment shown in Fig. 14 also verifies it feasible to offer voltage support for the AC-side loads and increase system faults tolerance.

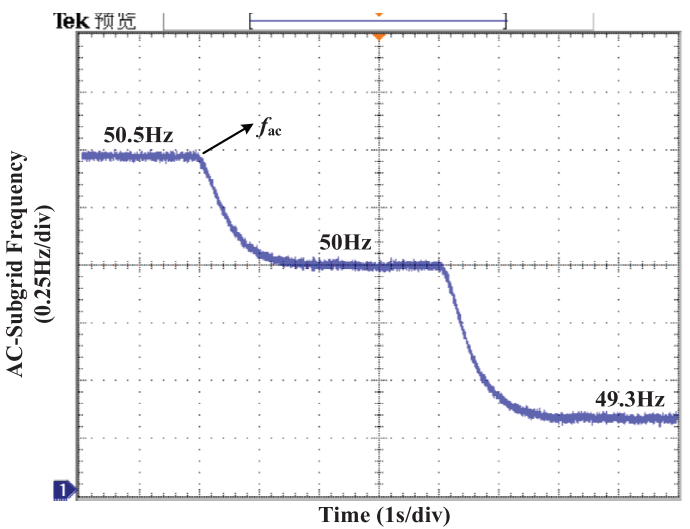

(a) AC-side frequency

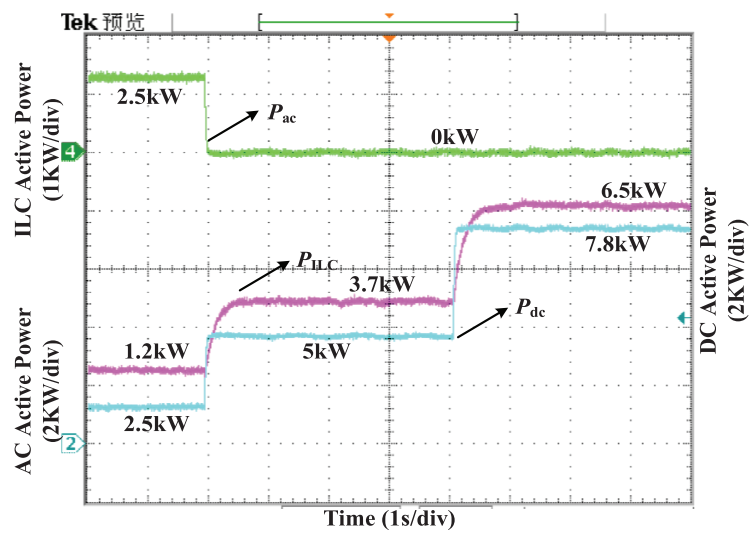

(b) Active power

Fig. 14. Waveforms of hybrid microgrid with AC subgrid faults events.

\section{CONCLUSION}

This paper proposes an improved wireless ILCs control strategy with synchronous generator characteristic for hybrid mcicrogrid. It aims to solve the problem of poor dynamic frequency stability as well as power oscillation during ACside load transients. Compared to conventional normalized droop control method, this strategy adopts direct frequency control to realize proportional power sharing and apply synchronverter technology to ILCs to increase system inertia of microgrids and damp AC bus frequency oscillations. Besides, exchanging power with voltage-type synchronverter technology could also offer uninterrupted power supply to the extreme AC-side loads during AC subgrid faults events. Finally, experiment results based on OPAL-RTLAB platform verify that this modified method can greatly improve the frequency transient stability and manage proportional power sharing meanwhile.

\section{REFERENCES}

[1] S. M. Malik, A. Xin, Y. Sun, Z. Chen, and S. Zhou,"Voltage and frequency control strategies of hybrid AC/DC microgrid: A review," IET Generation, Transmission \& Distribution, vol. 11, no. 2, pp. 303-313, Jan. 2017.

[2] F. Nejabatkhah and Y. W. Li, “Overview of power management strategies of hybrid AC/DC microgrid," IEEE Transactions on Power Electronics, vol. 30, no. 12, pp. 7072-7089, Dec. 2015.

[3] M. N. Ambia, A. Al-Durra, and S. M. Muyeen,"Centralized power control strategy for AC-DC hybrid micro-grid system using multi-converter scheme," in IECON 2011 - 37th Annual Conference of the IEEE Industrial Electronics Society, Melbourne, VIC, 2011, pp. 843-848.

[4] A. Mohamed, V. Salehi, and O. Mohammed,"Real-time energy management algorithm for mitigation of pulse loads in hybrid microgrids," IEEE Transactions on Smart Grid, vol. 3, no. 4, pp. 1911-1922, Dec. 2012.

[5] M. Hosseinzadeh and F. Salmasi,"Robust optimal power management system for a hybrid AC/DC micro-grid," IEEE Transactions on Sustainable Energy, vol. 6, no. 3, pp. 675-687, Jul. 2015.

[6] P. C. Loh, D. Li, Y. K. Chai, and F. Blaabjerg,"Autonomous operation of hybrid microgrid with AC and DC subgrids," IEEE Transactions on Power Electronics, vol. 28, no. 5, pp. 2214-2223, May 2013.

[7] V. K. Hema and R. Dhanalakshmi,"Power sharing of an autonomous hybrid microgrid with an interlinking converter," in IET Chennai Fourth International Conference on Sustainable Energy and Intelligent Systems (SEISCON 2013), Chennai, 2013, pp. 481-485.

[8] P. C. Loh, D. Li, Y. K. Chai, and F. Blaabjerg,"Autonomous control of interlinking converter with energy storage in hybrid AC-DC microgrid," IEEE Transactions on Industry Applications, vol. 49, no. 3, pp. 1374-1382, May-Jun. 2013.

[9] V. K. Hema and R. Dhanalakshmi, "Operation of hybrid AC-DC microgrid with an interlinking converter," in 2014 IEEE International Conference on Advanced Communications, Control and Computing Technologies, Ramanathapuram, 2014, pp. 38-42.

[10] W. Hu, H. Chen, X. Yang, K. Xu, and P. Hu, "Control strategy of the bi-directional converter for hybrid AC/DC microgrid," in 2015 IEEE PES Asia-Pacific Power and Energy Engineering Conference (APPEEC), Brisbane, QLD, 2015, pp. 1-5.

[11] J. Yu, W. Ming, L. Haitao, L. Yang, and Z. Ying,"Bidirectional droop control of interlinking converter in AC/DC hybrid micro-grid," in 20163 rd International Conference on Information Science and Control Engineering (ICISCE), Beijing, 2016, pp. 879-883.

[12] R. Majumder, B. Chaudhuri, A. Ghosh, R. Majumder, G. Ledwich, and F. Zare,"Improvement of stability and load sharing in an autonomous microgrid using supplementary droop control loop," in IEEE PES General Meeting, Minneapolis, MN, 2010, pp. 1-1.

[13] P. C. Loh and F. Blaabjerg, "Autonomous operation of hybrid microgrid with AC and DC sub-grids," in Proceedings of the 2011 14th European Conference on Power Electronics and Applications, Birmingham, 2011, pp. 1-10. 
[14] Q. C. Zhong and G. Weiss, "Synchronverters: Inverters that mimic synchronous generators," IEEE Transactions on Industrial Electronics, vol. 58, no. 4, pp. 1259-1267, Apr. 2011.

[15] Q. C. Zhong and G. Weiss,"Static synchronous generators for distributed generation and renewable energy," in 2009 IEEE/PES Power Systems Conference and Exposition, Seattle, WA, 2009, pp. 1-6.

[16] N. Xu, Y. Wang, M. Li, W. Wang, H. Wang, and H. Zhang,“Analysis and improvement of damping factor based on virtual synchronous generator control," in 2016 IEEE 8th International Power Electronics and Motion Control Conference (IPEMC-ECCE Asia), Hefei, 2016, pp. 1990-1996.

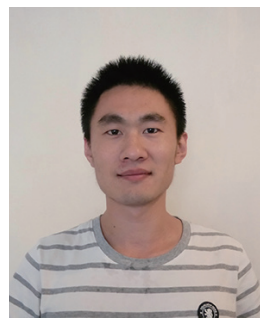

Gongxin Qi was born in Shandong, China, in1993. He received the B.S. degree from Shandong University, Jinan, China, in 2016, where he is currently working toward the M.S. degree in the Schoolof Control Science and Engineering.His current research is interlinking converters of hybrid AC/DC microgrid.

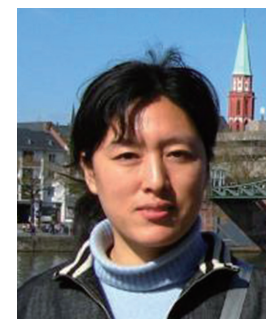

Alian Chen was born in Shandong, China, in 1976. She received the B.Sc. and M.Sc. degrees from Shandong University, Jinan, China, in 1998 and 2000, respectively, and the Ph.D. degree fromZhejiang University, Hangzhou, China, in 2005.In 2005, she joined the School of Control Scienceand Engineering, Shandong University. In 2013, she was a Visiting Scholar with the Center for Power Electronics Systems, Virginia Polytechnic Instituteand State University, Blacksburg, VA, USA. She has been involved in research on multilevel converters, power electronics, and industrial applications.

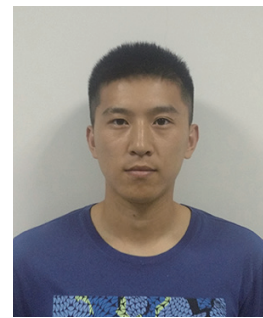

Jie Chen was born in China, in 1992. He received the B.S. degree in automation from the School of Control Science and Engineering, Shandong University, Jinan, China, in 2014, where he is current working towards the $\mathrm{Ph} . \mathrm{D}$ degree in power electronics. His current research interests include multi-level converters, power electronics for microgrid and dual-active bridge $\mathrm{dc} / \mathrm{dc}$ converter. 